\title{
Direct Experimental Determination of Grain Boundary Excess Volume in Metals
}

\author{
Eva-Maria Steyskal, Bernd Oberdorfer, and Wolfgang Sprengel* \\ Institute of Materials Physics, Graz University of Technology, A-8010 Graz, Austria \\ Michael Zehetbauer \\ Physics of Nanostructured Materials, Faculty of Physics, University of Vienna, A-1090 Vienna, Austria \\ Reinhard Pippan \\ Erich Schmid Institute of Materials Science, Austrian Academy of Sciences, and Department Materials Physics, \\ University of Leoben, A-8700 Leoben, Austria \\ Roland Würschum \\ Institute of Materials Physics, Graz University of Technology, A-8010 Graz, Austria
}

(Received 5 August 2011; published 31 January 2012)

\begin{abstract}
The grain boundary excess volume, i.e., the grain boundary expansion, $e_{\mathrm{GB}}$, was experimentally determined for high-angle grain boundaries in nickel using the direct technique of high-precision difference dilatometry. Values of $e_{\mathrm{GB}}=(0.35 \pm 0.04) \times 10^{-10} \mathrm{~m}$ and $e_{\mathrm{GB}}=(0.32 \pm 0.04) \times 10^{-10} \mathrm{~m}$ were obtained by measuring the removal of grain boundary volume upon grain growth for two different types of ultrafine-grained samples. The results are discussed in comparison to values obtained so far from indirect techniques and from computer simulations. It demonstrates the strength of the presented novel, direct approach for grain boundary expansion measurements.
\end{abstract}

Excess volume represents a key parameter in the physics of condensed matter. In amorphous solids, the structural disorder is usually accompanied by a reduced atomic density with excess volume being randomly distributed in the entire volume. In crystalline solids excess volume is localized at crystal defects, i.e., lattice vacancies, dislocations, and grain boundaries. Grain boundaries, homophase crystal-crystal interfaces, with disordered atomic structures are, therefore, much more complex than the periodic atomic structure of the crystallites they separate [1,2]. Grain boundaries with lower coordination and extended average bond length significantly determine the physical properties of polycrystalline solids [3-5]. It is especially the case for nanocrystalline materials where a large fraction of atoms is located in grain boundaries [6]. Despite their importance, grain boundaries are by far less well characterized than ordered bulk crystals. One fundamental key parameter for the characterization of a grain boundary is its volume expansion, i.e., the amount of its excess volume. The volume expansion is directly related to the grain boundary energy [7] and also significantly influences structural phenomena such as grain boundary diffusion and segregation [8] or physical phenomena such as the contribution of the grain boundary to the

Published by the American Physical Society under the terms of the Creative Commons Attribution 3.0 License. Further distribution of this work must maintain attribution to the author(s) and the published article's title, journal citation, and DOI. electrical resistivity [7]. This Letter presents, in a case study on nickel, the first, direct experimental determination of the excess volume of grain boundaries. It employs the macroscopic measurement of the irreversible length change of ultrafine-grained samples due to the release of grain boundary volume upon grain growth [9].

There have been efforts so far to determine the excess volume of grain boundaries experimentally, i.e., on isolated grain boundaries of known orientation by highresolution electron microscopy [10] which is a challenging task because the additional expansion is only a very small fraction of the lattice constant. Furthermore, as this technique relies on phase and image contrast it is by no means straightforward [11] and often applicable only to selected, well-defined tilt or twin boundaries. A different experimental approach reported recently by Shvindlerman et al. [12] utilizes the change of the grain boundary equilibration angle as function of hydrostatic pressure on tricrystals with a well-prepared orientation relation. This approach relies on appropriate thermodynamic grain boundary modeling.

In polycrystalline, single-phase solids, grain boundaries represent internal interface areas between regions of different crystallographic orientation. Because of the mismatch of the crystal lattice at the grain boundary, the total number of atoms, $n_{i}$, in a polycrystalline solid occupy more volume than would be the case if the atoms are arranged in a perfect single crystal. If no other lattice defects such as vacancies or dislocations are present, then the grain boundary expansion of relaxed grain boundaries can be determined from density changes or, which is equivalent, from 
the change of the macroscopic volume due to the removal of the grain boundaries as a consequence of grain growth. The expansion of the grain boundary $e_{\mathrm{GB}}$ is then defined as the change of the volume $V$ with the grain boundary area $A$,

$$
e_{\mathrm{GB}}=\left(\frac{\partial V}{\partial A}\right)_{T, p, n_{i}}
$$

at constant temperature $T$ and pressure $p$, and for a constant number $n_{i}$ of atoms. It has the unit of a length $\left[\mathrm{m}^{3} / \mathrm{m}^{2}\right]$. The grain boundary expansion is an excess physical property with respect to the ideal, perfectly ordered crystal and its value can be as low as $1 / 10$ of the lattice constant or even lower, e.g., in the case of twin boundaries. The grain boundary expansion $e_{\mathrm{GB}}$ should not be confused with the physical grain boundary width $\delta_{\mathrm{GB}}$ that is usually of the order of 0.5 to $1 \mathrm{~nm}$ [13], thus, at least 1 order of magnitude larger.

For the present study, high-precision differencedilatometric measurements were used to determine the release of the excess grain boundary volume upon annealing. It makes use of the fact that due to grain growth from an initial grain size $d_{\text {ini }}$ to a final grain size $d_{\text {fin }}$, the release of the excess volume of the grain boundary can be measured by the length change $\Delta L$ of a specimen with length $L_{0}$ according to

$$
\left.\frac{\Delta L}{L_{0}}\right|_{\mathrm{ini}}-\left.\frac{\Delta L}{L_{0}}\right|_{\mathrm{fin}}=e_{\mathrm{GB}}\left(\frac{1}{d_{\mathrm{ini}}}-\frac{1}{d_{\mathrm{fin}}}\right),
$$

where $e_{\mathrm{GB}}$ is the grain boundary expansion, and the $\Delta L / L_{0}$ values are the net length changes determined as difference to a coarse-grained, defect-free sample of the same material. If the grain shape is anisotropic, $d_{\text {ini }}$ and $d_{\text {fin }}$ denote the distances between opposite grain boundaries with respect to the measuring direction. The applied technique is an integral one. The accuracy of the $e_{\mathrm{GB}}$ value is mainly determined by the initial value of $d_{\text {ini }}$. The effect is most drastic for a solid with a high concentration of interfaces. Therefore, nanocrystalline or ultrafine-grained materials are most suitable.

An ultrafine-grained nickel specimen disc with a diameter of $30 \mathrm{~mm}$ and a height of $10 \mathrm{~mm}$ was prepared by the severe plastic deformation (SPD) technique of high-pressure torsion (HPT) from high-purity nickel $(99.99+\%)$. A total of 5 revolutions applying a pressure of $2.2 \mathrm{GPa}$ were carried out for the grain refinement. The specimen disc in the as-prepared state is $100 \%$ dense and free of cracks and pores [14]. Samples were cut from the disc from a region with a radius larger than $5 \mathrm{~mm}$ from the center. In this region a homogeneous plastic deformation in saturation has occurred and the microstructure is homogenous and independent of the radius and strain. Because of the parameter selection of the high-pressure torsion process the obtained ultrafine-grained microstructure showed an anisotropic grain orientation with ellipsoidal shaped grains as revealed by scanning electron microscopy
(SEM). The aspect ratio of the grains was determined to be between 1.8 and 1.7. Two types of specimens, in total 15 , prism-shaped samples with a nominal size of $3 \mathrm{~mm} \times$ $3 \mathrm{~mm} \times 7 \mathrm{~mm}$ were prepared. One type showed elongated grain axes parallel to the long sample axis (3 samples) and for one type the elongated grain axes were perpendicular to the long sample axis (12 samples). For the dilatometric measurements all samples were positioned upright, with the long sample axis in the direction of the dilatometric measurement. A high-precision, vertical difference dilatometer (Linseis L75VD500LT) was employed which allows two independent length change measurements at the same time. Measurements were performed under pure $\mathrm{Ar}$ $(99.999 \%)$ gas flow and with reference to a well-annealed, coarse-grained, and defect-free nickel specimen in thermodynamic equilibrium prepared from the same specimen disc.

The top graph in Fig. 1 shows an example of a dilatometric curve, i.e., the relative length change $\Delta L / L_{0}$, of an ultrafine-grained sample measured as difference curve with the well-annealed, coarse-grained Ni reference. The length change has been measured with a linear heating rate of $6 \mathrm{~K} / \mathrm{min}$ in the temperature range from 293 to $673 \mathrm{~K}$. Two major stages, (A) and (B), can be identified that are relevant for the analysis. Stage (A) occurs from $293 \mathrm{~K}$ up to about $470 \mathrm{~K}$. As a result from recent in situ positron annihilation measurements on the very same sample material by the authors [15] this stage is attributed to annealing of vacancies and a significant reduction in the dislocation density that were introduced during the HPT process. The finding that by annealing HPT deformed $\mathrm{Ni}$ samples up to $470 \mathrm{~K}$ besides vacancies also the number of dislocations is drastically decreased is supported by results from X-ray measurements of other, independent studies $[16,17]$. Furthermore, during stage (A) also relaxation of grain boundaries and removal of micro- and macrostrains take place.

Within the temperature interval of stage (A) from 293 to $470 \mathrm{~K}$, the mean grain size of the sample does not change significantly as was confirmed by image analyses of micrographs obtained from SEM. However, due to a rearrangement of dislocations in this stage the aspect ratio of the grains is slightly reduced to 1.7. Detailed image analyses of SEM micrographs after the first stage (A), indicated by (1) in Fig. 1, showed a mean grain boundary distance of $(298 \pm 4) \mathrm{nm}$ in the direction of the long sample axis for the specimens with elongated grains parallel to the long sample axis, and $(175 \pm 4) \mathrm{nm}$ for the specimens with the elongated grains perpendicular to the long sample axis.

In the succeeding stage (B) at higher temperature $(T>470 \mathrm{~K})$ the defects remaining in the samples at the beginning of stage (B) are predominantly relaxed grain boundaries and the length change is only caused by the removal of these grain boundaries due to grain growth (see 

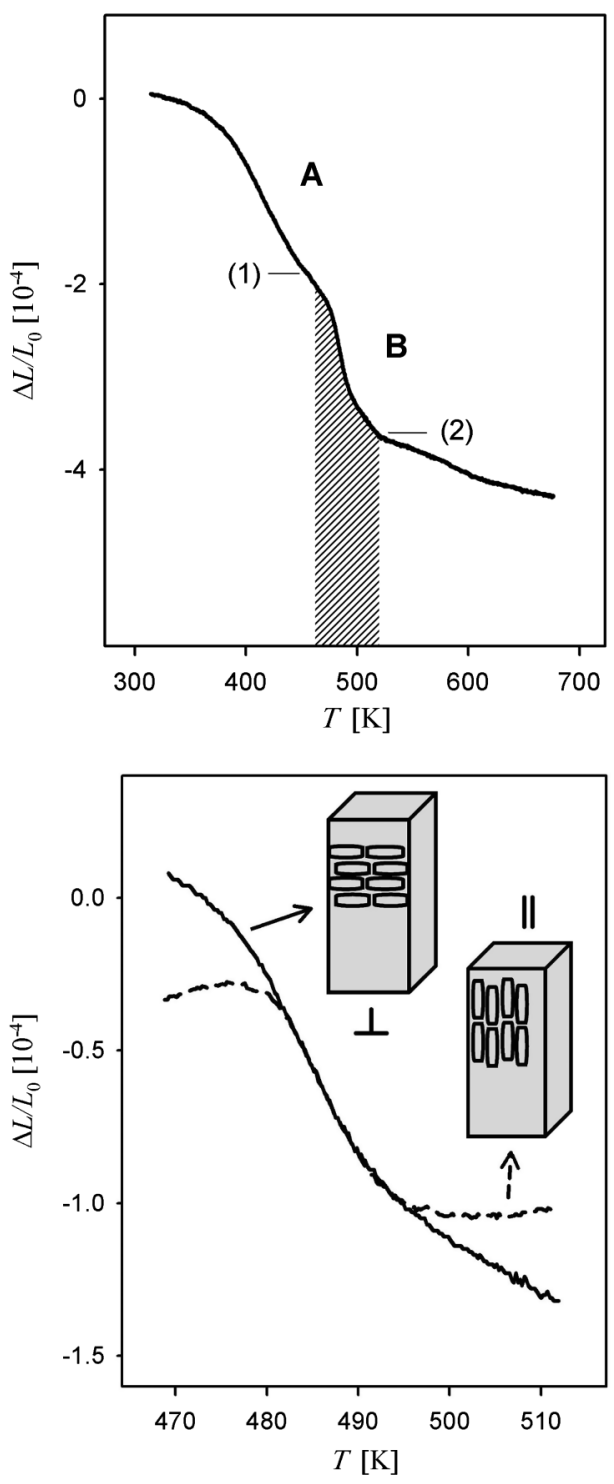

FIG. 1. Temperature dependence of the relative length change $\Delta L / L_{0}$ of ultrafine-grained Ni samples. Top: Annealing of free volume comprising annealing of vacancies and rearrangement of dislocations as well as grain boundary relaxation in a first stage denoted by (A), and grain growth in a second stage at higher temperatures denoted by (B). Bottom: Focus on the relative length change in the stage (B) of grain growth, in the temperature range from 470 to $510 \mathrm{~K}$ for two different grain orientations with respect to the measuring direction (for details see text). The starting point $\left(\Delta L / L_{0}\right)_{\text {ini }}$ and the ending point $\left(\Delta L / L_{0}\right)_{\text {fin }}$ of stage (B) are indicated in the top graph by (1) and by (2), respectively.

Fig. 1, bottom). For this case study nickel has the advantage that the two stages, stage (A) with annealing of vacancies and rearrangement of dislocations, and stage (B) the grain growth stage, can be clearly separated; i.e., the former processes do not overlap with the latter one [15]. Thus, after appropriate heat treatment during stage (A) a microstructure without significant loss of grain boundaries consists mainly of relaxed high-angle grain boundaries. Their internal structure is characteristic for conventional grain boundaries found, e.g., in coarsegrained material. These findings were shown experimentally $[18,19]$ as well as by computer simulation, see, e.g., Ref. [20]. Without loss of generality, ultrafine-grained materials of this type are therefore suitable for the assessment of the grain boundary expansion, $e_{\mathrm{GB}}$.

Then stage (B) is attributed to a grain growth process as is confirmed by SEM image analyses of samples taken after the occurrence of stage (B). The exact beginning, $\left(\Delta L / L_{0}\right)_{\text {ini }}$, and end $\left(\Delta L / L_{0}\right)_{\text {fin }}$ of stage (B) are crucial points for the determination of the value of the grain boundary expansion as well as for the grain size analyses by SEM that were taken at the exact same conditions. As seen from Fig. 1 (top) the two stages (A) and (B) can clearly be distinguished by their distinct curvature. Therefore, extrema of the first derivative of the dilatometric curves with respect to temperature, $\partial\left(\Delta L / L_{0}\right) / \partial T$, were used to unambiguously determine the starting and ending points of the stage (B) for the 15 samples. The lower part of Fig. 1 shows examples for two samples, one with the elongated grain axis parallel ( II) and one with the elongated grain axis perpendicular $(\perp)$ to the measurement direction. Form the two graphs it is also clear to notice that the length change effect of grain growth is different for the two different grain orientations. As expected, it is smaller for the samples of grain orientation with elongated axes parallel to the measuring directions as less grain boundaries contribute to the length change in that direction.

For all measured samples the length change contributions of stage (B) due to grain growth were determined for the two orientations. In Table I their average values $\Delta L / L_{0}$ are given. From these data according to Eq. (2) values for the grain boundary expansion of $\mathrm{Ni}$ were determined with respect to both grain orientations. For the case of elongated grain axis perpendicular to the expansion direction $(\perp)$ a value of $e_{\mathrm{GB}}=(0.35 \pm 0.04) \times 10^{-10} \mathrm{~m}$ is obtained. In the case where the elongated grain axis is parallel ( $\|$ ) the result is a value of $e_{\mathrm{GB}}=(0.32 \pm 0.04) \times 10^{-10} \mathrm{~m}$. As in total 12 samples with $(\perp)$ orientation and 3 samples with ( II ) orientation have been analyzed the values for both orientations represent the averaged values, respectively. The uncertainty ranges of the values are derived from the standard deviation of the in total 12 and 3 single values. The results of the measurement for the two independent grain orientations give the same values within the range of uncertainty. It confirms the reliability of this analysis method. Moreover, the identical values for $e_{\mathrm{GB}}$ for both orientations clearly confirm that the observed irreversible length change $\Delta L / L_{0}$ is solely due to GB excess free volume since the difference of $\Delta L / L_{0}$ for both orientations can quantitatively be associated with the different numbers of grain boundaries. 
TABLE I. Values for the absolute grain boundary expansion, $e_{\mathrm{GB}}$, and values $e_{\mathrm{GB}} / a$ normalized to the lattice constant (Ni: $a=$ $3.533 \times 10^{-10} \mathrm{~m}$ at $\left.470 \mathrm{~K}[21]\right)$. The values $d_{\text {ini }}$ denote the initial and $d_{\text {fin }}$ the final grain dimension, i.e., the distances between opposite grain boundaries with respect to the measuring direction. The values $\left(\Delta L / L_{0}\right)$ are the specific length change contributions of the grain growth stage B and calculated as difference of $\left(\Delta L / L_{0}\right)_{\text {ini }}-\left(\Delta L / L_{0}\right)_{\text {fin }}$ at the temperatures indicated in the figure by (1) and (2), respectively. All values are arithmetic mean values from $N$ different ultrafine-grained Ni samples measured by dilatometry. Two distinct grain orientations were used: elongated axis perpendicular, $\perp(N=12)$, or parallel, $\|(N=3)$, to the measurement direction.

\begin{tabular}{lccccc}
\hline \hline & $d_{\text {ini }}\left[10^{-9} \mathrm{~m}\right]$ & $d_{\text {fin }}\left[10^{-6} \mathrm{~m}\right]$ & $\Delta L / L_{0}\left[10^{-4}\right]$ & $e_{\mathrm{GB}}\left[10^{-10} \mathrm{~m}\right]$ & $e_{\mathrm{GB}} / a$ \\
\hline$\perp$ & $(175 \pm 4)$ & $(1.4 \pm 0.02)$ & $(1.76 \pm 0.20)$ & $(0.35 \pm 0.04)$ & $(0.10 \pm 0.01)$ \\
$\|$ & $(298 \pm 4)$ & $(1.4 \pm 0.02)$ & $(0.85 \pm 0.24)$ & $(0.32 \pm 0.04)$ & $(0.09 \pm 0.01)$ \\
\hline \hline
\end{tabular}

The $e_{\mathrm{GB}}$ value deduced in the present work by dilatometry upon grain growth represents the value at the temperature where the first grain growth sets in. For the present case of nickel measured with a linear heating rate of $6 \mathrm{~K} / \mathrm{min}$ this occurs at about $470 \mathrm{~K}$. As the sample is measured with reference to a well-annealed coarse-grained sample, the contribution of the reversible linear thermal expansion of the crystal has no effect on the difference curve. However, the contribution of the linear thermal expansion of the grain boundary, i.e., the thermal expansion coefficient $\alpha^{\mathrm{GB}}$, has to be considered if, for comparison, the room temperature value of the grain boundary expansion is to be calculated. Birringer et al. [22] determined thermal expansion coefficients from dilatometric measurements on nanocrystalline palladium. They found that the thermal expansion coefficient of relaxed grain boundaries in palladium is only by a factor of 1.1 higher than the thermal expansion coefficient $\alpha^{\text {cryst }}$ of the crystal $\left(\alpha^{\mathrm{GB}}=1.1 \times \alpha^{\text {cryst }}\right)$. Employing a rule of mixture for the total reversible thermal expansion coefficient for the nanocrystal $\alpha^{\mathrm{nc}}=(1-x) \alpha^{\text {cryst }}+x \alpha^{\mathrm{GB}}$ and taking into account that for the present case the grain boundary fraction, $x$, is less than $1 \%$ then for a physical grain boundary width $\delta$ of $0.5 \mathrm{~nm}$ and a temperature increase by $170 \mathrm{~K}$ the contribution to the value of $e_{\mathrm{Gb}}$ is by far less than $1 \mathrm{pm}$ and, thus, is negligible.

Now an estimation on the absolute uncertainty of the value of the grain boundary expansion is given. One uncertainty might be caused by the removal of remnant dislocations from the microstructure during the grain growth stage (B). Assuming a value of an initial dislocation density $\rho$ in the as-prepared state of $3 \times 10^{15} / \mathrm{m}^{2}$ as has been reported for HPT deformed Ni [16], such a dislocation density would contribute an amount of $\Delta L / L=3.1 \times$ $10^{-5}(18 \%)$ to the length change value if for the excess volume of a dislocation and the corresponding length change the textbook relation $\Delta L / L_{0}=1 / 3 \times \Delta V / V_{0}=$ $0.5 \times b^{2} \times \rho$ is used [9], and where $b$ denotes the length of the Burgers vector. However, the value of the dislocation density in SPD deformed Ni is lowered upon annealing up to $470 \mathrm{~K}$ by at least 1 order of magnitude as, e.g., reported in Ref. [17]. Therefore, annealing of remnant dislocations during the grain growth process likely contributes only a few percent to the value of the grain boundary expansion volume as reported in the present study.
Another uncertainty might arise from the conversion of grain boundary volume into vacancies during grain growth as reported in a model by Estrin et al. [23]. Assuming reasonable vacancy diffusion data for pure Ni from literature [24] these vacancies would anneal out at sinks in a range of $100 \mathrm{~nm}$ within seconds at temperatures above $470 \mathrm{~K}$. The grain growth process reported here for a heating rate of $6 \mathrm{~K} / \mathrm{min}$ in the temperature interval from 470 to $510 \mathrm{~K}$ occurs on a time scale which is at least 1 order of magnitude longer. Therefore, remnant single vacancies emitted from migrating grain boundaries during grain growth are unlikely. In the case that emitted vacancies form vacancy clusters or pores removable only at higher temperature they would contribute less than $20 \%$ as deduced from the mean length change measured for all 15 samples after the grain growth stage up to $680 \mathrm{~K}$. In this sense the reported values of $0.35 \times 10^{-10}$ and $0.32 \times$ $10^{-10}$ can be seen as a lower bound.

Formation of an interconnected network of free-volume channels as has been reported by Divinsky et al. [25] for nanocrystalline $\mathrm{Cu}$ and $\mathrm{Ni}$ can be ruled out as source of uncertainty. A volume fraction $\Delta V / V$ for these defects in nanocrystalline $\mathrm{Ni}$ severely deformed by equal-channel angular pressing was estimated to amount to about 0.1 ppm only [25]; i.e., $\Delta L / L=3 \times 10^{-8}$. Such a small value is negligible for the observed length changes in the $10^{-4}$ range of the present study.

For the grain boundary expansion, only few experimental data are available in the literature, e.g., for $\mathrm{Au}, e_{\mathrm{GB}}=0.04$ to $0.10 \times 10^{-10} \mathrm{~m}$ [10] or $e_{\mathrm{GB}}=0.12 \times 10^{-10} \mathrm{~m}$ [26] from high-resolution transmission electron microscopy. Shvindlerman et al. [12] experimentally determined the excess grain boundary volume in pure aluminum using a thermodynamic approach employing the pressure dependence of a grain boundary contact angle in a tricrystal. They found $e_{\mathrm{GB}}=0.64 \times 10^{-10} \mathrm{~m}$ for Al. The above data were mostly obtained on isolated grain boundaries with distinct orientation relation. For nanocrystalline $\mathrm{Pd}$ a value of $e_{\mathrm{GB}}=0.23 \times 10^{-10} \mathrm{~m}$ was determined from density measurements [22]. And for nanocrystalline Fe, $e_{\mathrm{GB}}=0.19 \times$ $10^{-10} \mathrm{~m}$ [27] was derived from modeling of grain growth kinetics. Data sets of grain boundary expansion are also available from computer simulations [28-31]. Though simulation techniques nowadays are quite powerful and allow for simulation of complex atomic structures such as 
grain boundaries, the results, however, are sensitively dependent of the choice of the interatomic potentials [28]. Most recently grain boundary expansion data have been reported from molecular dynamic simulations on nickel $\Sigma 5$ grain boundaries $e_{\mathrm{GB}}=0.39$ to $0.41 \times 10^{-10} \mathrm{~m}$ (at $T=$ $1200 \mathrm{~K})$ [30], and nickel high-angle grain boundaries $e_{\mathrm{GB}}=0.28$ to $0.42 \times 10^{-10} \mathrm{~m}$ [31]. Here, the matching of the data values with the data of the present study is remarkable. Much higher values of $e_{\mathrm{GB}}=1.6 \times 10^{-10} \mathrm{~m}$ have been reported recently that were obtained from x-ray diffraction of stressed, nanocrystalline Ni thin films [32].

The experimental approach presented in this Letter is quite powerful as it is a direct and integral method for determining the grain boundary expansion from macroscopic dilatometric measurements on nanocrystalline samples. As demonstrated in this study a physical key parameter of grain boundaries, i.e., the grain boundary excess volume, can be determined in a direct and straightforward manner.

Financial support by Austrian Science Fund (FWF): P21009-N20 is appreciated.

*w.sprengel@tugraz.at

[1] A. P. Sutton and R. W. Balluffi, Interfaces in Crystalline Materials (Oxford University Press, Oxford, 1995).

[2] A. Chua, N. Benedek, A. Nicole, L. Chen, M. Finnis, and A. Sutton, Nature Mater. 9, 418 (2010).

[3] R. Z. Valiev, R. K. Islamgaliev, and I. V. Alexandrov, Prog. Mater. Sci. 45, 103 (2000).

[4] R.Z. Valiev, Nature Mater. 3, 511 (2004).

[5] Bulk Nanostructured Materials, edited by M.J. Zehetbauer and Y. T. Zhu (Wiley VCH, Weinheim, 2009).

[6] H. Gleiter, Acta Mater. 48, 1 (2000).

[7] A. Seeger and G. Schottky, Acta Metall. 7, 495 (1959).

[8] H. Mehrer, Diffusion in Solids (Springer, New York, 2007).

[9] B. Oberdorfer, B. Lorenzoni, K. Unger, W. Sprengel, M. Zehetbauer, R. Pippan, and R. Würschum, Scr. Mater. 63, 452 (2010).

[10] K. L. Merkle, Ultramicroscopy 40, 281 (1992).

[11] J. M. Pennisson and T. Vystavel, Ultramicroscopy 90, 163 (2002).
[12] L. S. Shvindlerman, G. Gottstein, V. A. Ivanov, D. A. Molodov, D. Kolesnikov, and W. Lojkowski, J. Mater. Sci. 41, 7725 (2006).

[13] I. Kaur, Y. Mishin, and W. Gust, Fundamentals of Grain and Interphase Boundary Diffusion (Wiley, Chichester, 1995), 3rd ed.

[14] R. Pippan, S. Scheriau, A. Taylor, M. Hafok, A. Hohenwarter, and A. Bachmaier, Annu. Rev. Mater. Res. 40, 319 (2010).

[15] B. Oberdorfer, E.-M. Steyskal, W. Sprengel, W. Puff, P. Pikart, C. Hugenschmidt, M. Zehetbauer, R. Pippan, and R. Würschum, Phys. Rev. Lett. 105, 146101 (2010).

[16] E. Schafler and R. Pippan, Mater. Sci. Eng. A 387-389, 799 (2004).

[17] Z. Q. Yang, Mater. Lett. 60, 3846 (2006).

[18] H.-E. Schaefer, K. Reimann, W. Straub, F. Phillipp, H. Tanimoto, U. Brossmann, and R. Würschum, Mater. Sci. Eng. A 286, 24 (2000).

[19] R. Würschum, S. Herth, and U. Brossmann, Adv. Eng. Mater. 5, 365 (2003).

[20] H. Van Swygenhoven, D. Farkas, and A. Caro, Phys. Rev. B 62, 831 (2000).

[21] Handbook of Chemistry and Physics, edited by D. R. Lide (CRC Press, Taylor \& Francis, Boca Raton, 2006), 87th ed.

[22] R. Birringer, C.E. Krill, and M. Klingel, Philos. Mag. Lett. 72, 71 (1995)

[23] Y. Estrin, G. Gottstein, E. Rabkin, and L. Shvindlerman, Scr. Mater. 43, 141 (2000).

[24] Atomic Defects in Metals-Landolt-Börnstein-New Series/ Condensed Matter, edited by H. Ullmaier (Springer, Berlin, Heidelberg, 1991), Vol. 23.

[25] S. V. Divinski, G. Reglitz, H. Rösner, Y. Estrin, and G. Wilde, Acta Mater. 59, 1974 (2011).

[26] M. I. Buckett and K. L. Merkle, Ultramicroscopy 56, 71 (1994).

[27] C. E. Krill, L. Helfen, D. Michels, H. Natter, A. Fitch, O. Masson, and R. Birringer, Phys. Rev. Lett. 86, 842 (2001).

[28] D. Wolf, Scr. Metall. 23, 1913 (1989).

[29] D. Wolf, Philos. Mag. A 62, 447 (1990).

[30] H. Zhang and D. J. Srolovitz, Acta Mater. 54, 623 (2006).

[31] D. L. Olmsted, S. M. Foiles, and E. A. Holm, Acta Mater. 57, 3694 (2009).

[32] Y. Kuru, M. Wohlschlögel, U. Welzel, and E. J. Mittemeijer, Appl. Phys. Lett. 95, 163112 (2009). 